Pacific Journal of Mathematics

CHARACTERIZATIONS OF CONDITIONAL 


\title{
CHARACTERIZATIONS OF CONDITIONAL EXPECTATION-TYPE OPERATORS
}

\author{
P. G. Dodds, C. B. Huijsmans and B. De Pagter
}

A complete description of positive projections in ideals of measurable functions is given in terms of conditional expectation-type operators.

1. Introduction. As is well-known, conditional expectation operators on various function spaces exhibit a number of remarkable properties related either to the underlying order structure of the given function space, or to the metric structure when the function space is equipped with a norm. Such operators are necessarily positive projections which are averaging in a precise sense to be described below and in certain normed function spaces are contractive for the given norm. Conditional expectation operators on $L^{p}$-spaces have been characterized in terms of their averaging property by Moy [M] and Rota [Ro] and as contractive projections by Douglas [D1] and Ando [A].

More recently, positive projections of a certain class of Banach function spaces have been characterized in terms of conditional expectation by Kulakova [Ku]. It is our intention in this paper to show that the characterization of positive projections given by Kulakova remains valid for arbitrary ideals of measurable functions. The method of [Ku] is based on the approach of Douglas for the case of $L^{1}$-spaces and makes essential use of the underlying metric structure via an appeal to a well-known interpolation theorem for rearrangement invariant KB-spaces. The approach to the present paper is on the other hand purely algebraic and uses the underlying order structure via a suitable adaptation of the ideas of Moy on averaging operators. The basic link which in essence reduces the more general problem of describing positive projections to the study of averaging operators is provided by a result of Kelley [K] which implies that each positive projection on an $L^{\infty}$-space with range a vector sublattice containing the constants is necessarily averaging.

After gathering some preliminary notions in $\S 2$, we consider in $\S 3$ the relation between conditional expectations and averaging operators 
in the setting of ideals of measurable functions. The characterization of averaging operators, given in Proposition 3.1 and inspired by the work of Moy, is in turn an extension of her result. The connection with positive projections via the Kelley theorem is given in Proposition 3.3. The principal result of the paper is Theorem 3.10, which extends the work of Kulakova and which yields a complete description of positive order continuous projections in terms of (so-called) weighted conditional expectation operators. This theorem implies, in particular, that each strictly positive order continuous projection for which the constant functions are invariant is of conditional expectation type. We indicate in $\S 4$ the extent to which the present approach yields characterizations of conditional expectation on Banach function spaces which extend those of Rota for averaging contractions and of Ando and Douglas for positive contractive projections on $L^{p}$-spaces. In general, satisfactory extensions are valid only under additional smoothness assumptions which are automatically satisfied in the $L^{p}$-setting. Related results may be found in the thesis of Duplissey [Du] and in the papers of Rao [Ra1], [Ra2]. However, results from this latter source should be treated with some caution, as has been pointed out explicitly by Bernau and Lacey [BL]. Finally, we show that each contractive projection in a Banach function space with range a sublattice containing the constant functions is necessarily positive, provided a certain smoothness assumption is satisfied (Proposition 4.13). While this smoothness assumption is again necessarily satisfied in the case of $L^{p}$-spaces, Example 4.11 shows that it cannot be omitted even in the finite dimensional setting.

2. Preliminaries. We assume that the reader is familiar with the basic concepts of the theory of vector lattices and Banach lattices (Banach function spaces). For unexplained terminology and notations we refer to the books $[\mathbf{A B}],[\mathbf{L Z}],[\mathbf{S c h}]$ and $[\mathrm{Z}]$. The main purpose of this section is to fix some of the notation and terminology to be used in the present paper. Throughout this paper, $(\Omega, \Sigma, \mu)$ is a finite measure space. If $\Sigma_{0}$ is a $\sigma$-subalgebra of $\Sigma$, then we denote the restriction of $\mu$ to $\Sigma_{0}$ (abusively) by $\mu$ again. The characteristic function of a set $A \in \Sigma$ is denoted by $\mathbf{1}_{A}$. However, we write 1 rather than $\mathbf{1}_{\Omega}$. For any measurable function $f$ on $\Omega$ the support of $f$ is denoted by $\operatorname{supp}(f)=\{\omega \in \Omega: f(\omega) \neq 0\}$. We shall consider only (extended) real-valued functions in this paper, as all the results extend without difficulty to the complex case. 
The space of all real valued $\Sigma$-measurable functions on $\Omega$, with the usual identification of $\mu$-a.e. equal functions, is denoted by $L^{0}(\Sigma, \mu)$. Similarly, we write $L^{p}(\Sigma, \mu)$ for the corresponding $L^{p}$-spaces $(1 \leq p \leq$ $\infty)$. By an ideal of measurable functions on $(\Omega, \Sigma, \mu)$ we mean a linear subspace $E$ of $L^{0}(\Sigma, \mu)$ such that $g \in E, f \in L^{0}(\Sigma, \mu),|f| \leq|g|$ implies $f \in E$. We shall always assume that $L^{\infty}(\Sigma, \mu) \subseteq E \subseteq L^{1}(\Sigma, \mu)$. For such an ideal $E$ the associate space $E^{\prime}$ consists of all $g \in L^{0}(\Sigma, \mu)$ for which $\int_{\Omega}|f g| d \mu<\infty$ for all $f \in E$. Clearly, $E^{\prime}$ is an ideal of measurable functions on $(\Omega, \Sigma, \mu)$. It is clear that any $g \in E^{\prime}$ defines a linear functional $\varphi_{g}$ on $E$ given by $\left\langle f, \varphi_{g}\right\rangle=\int_{\Omega} f g d \mu$ for all $f \in E$. If, in addition, there is a norm $\|\cdot\|_{E}$ in the ideal $E$ which is absolutely monotone (i.e., $|f| \leq|g|$ in $E$ implies $\left.\|f\|_{E} \leq\|g\|_{E}\right)$ such that $\left(E,\|\cdot\|_{E}\right)$ is a Banach space, then $\left(E,\|\cdot\|_{E}\right)$ is called a Banach function space. Note that in this case the inclusions $L^{\infty}(\Sigma, \mu) \subseteq E \subseteq L^{1}(\Sigma, \mu)$ imply (by the closed graph theorem) that $c\|\cdot\|_{1} \leq\|\cdot\|_{E} \leq C\|\cdot\|_{\infty}$ for some constants $c, C>0$. Furthermore, for all $g \in E^{\prime}$ we have $\varphi_{g} \in E^{*}$, the Banach dual of $E$. The mapping $g \mapsto \varphi_{g}$ is an embedding of $E^{\prime}$ into $E^{*}$. Henceforth, we shall consider $E^{\prime}$ as a subspace of $E^{*}$. As is well known, $E^{*}=E^{\prime}$ if and only if $\|\cdot\|_{E}$ is order continuous.

Next we collect some simple properties of conditional expectations. Suppose that $\Sigma_{0}$ is a $\sigma$-subalgebra of $\Sigma$. For any $f \in L^{1}(\Sigma, \mu)$ we denote by $\mathscr{E}^{\mu}\left(f \mid \Sigma_{0}\right)$ the ( $\mu$-a.e. $)$ unique $\Sigma_{0}$-measurable function with the property that

$$
\int_{A} f d \mu=\int_{A} \mathscr{E}^{\mu}\left(f \mid \Sigma_{0}\right) d \mu
$$

for all $A \in \Sigma_{0}$. The existence of $\mathscr{E}^{\mu}\left(f \mid \Sigma_{0}\right)$ is an easy consequence of the Radon-Nikodym theorem. The function $\mathscr{E}^{\mu}\left(f \mid \Sigma_{0}\right)$ is called the conditional expectation of $f$ with respect to $\Sigma_{0}$. If there is no possibility of confusion, we write $\mathscr{E}\left(f \mid \Sigma_{0}\right)$ instead of $\mathscr{E}^{\mu}\left(f \mid \Sigma_{0}\right)$. We list some elementary properties (see e.g. $[\mathbf{N}]$ ).

(i) $\mathscr{E}\left(\cdot \mid \Sigma_{0}\right)$ is a linear mapping from $L^{1}(\Sigma, \mu)$ into itself;

(ii) $\mathscr{E}\left(\cdot \mid \Sigma_{0}\right)$ is positive, i.e., $\mathscr{E}\left(f \mid \Sigma_{0}\right) \geq 0$ whenever $0 \leq f \in L^{1}(\Sigma, \mu)$;

(iii) $\mathscr{E}\left(f \mid \Sigma_{0}\right)=f$ if and only if $f \in L^{1}\left(\Sigma_{0}, \mu\right)$, so $\mathscr{E}\left(\cdot \mid \Sigma_{0}\right)$ is a projection from $L^{1}(\Sigma, \mu)$ onto $L^{1}\left(\Sigma_{0}, \mu\right)$; in particular $\mathscr{E}\left(\mathbf{1} \mid \Sigma_{0}\right)=\mathbf{1}$; furthermore $\mathscr{E}\left(f \mid \Sigma_{0}\right) \in L^{\infty}\left(\Sigma_{0}, \mu\right)$ whenever $f \in L^{\infty}(\Sigma, \mu)$;

(iv) if $f \geq 0$ then $\left\|\mathscr{E}\left(f \mid \Sigma_{0}\right)\right\|_{1}=\|f\|_{1}$ and by virtue of the positivity of $\mathscr{E}\left(\cdot \mid \Sigma_{0}\right)$ we have $\left|\mathscr{E}\left(f \mid \Sigma_{0}\right)\right| \leq \mathscr{E}\left(|f| \mid \Sigma_{0}\right)$, so that $\left\|\mathscr{E}\left(f \mid \Sigma_{0}\right)\right\|_{1} \leq\|f\|_{1}$ for all $f \in L^{1}(\Sigma, \mu)$. In other words, $\mathscr{E}\left(\cdot \mid \Sigma_{0}\right)$ is a contraction in $L^{1}(\Sigma, \mu)$; 
(v) $\mathscr{E}\left(\cdot \mid \Sigma_{0}\right)$ is order continuous, i.e., if $0 \leq f_{n} \uparrow f$ a.e. in $L^{1}(\Sigma, \mu)$ then $\mathscr{E}\left(f_{n} \mid \Sigma_{0}\right) \uparrow \mathscr{E}\left(f \mid \Sigma_{0}\right)$ a.e.;

(vi) $\mathscr{E}\left(\cdot \mid \Sigma_{0}\right)$ satisfies the averaging identity i.e. $\mathscr{E}\left(f \mathscr{E}\left(g \mid \Sigma_{0}\right) \mid \Sigma_{0}\right)=$ $\mathscr{E}\left(f \mid \Sigma_{0}\right) \mathscr{E}\left(g \mid \Sigma_{0}\right)$ for all $f \in L^{\infty}(\Sigma, \mu)$ and $g \in L^{1}(\Sigma, \mu)$.

Let $M^{+}(\Sigma, \mu)$ be the collection of all positive extended real valued $\Sigma$-measurable functions on $\Omega$ (with the usual identification). Using (v) it is easy to see that $\mathscr{E}\left(\cdot \mid \Sigma_{0}\right)$ can be extended uniquely to an order preserving, additive, positive homogeneous mapping from $M^{+}(\Sigma, \mu)$ into itself, which still satisfies (v) and also the averaging identity (vi) for all $f, g \in M^{+}(\Sigma, \mu)$.

As before, let $E$ be an ideal of measurable functions on the measure space $(\Omega, \Sigma, \mu)$ such that $L^{\infty}(\Sigma, \mu) \subseteq E \subseteq L^{1}(\Sigma, \mu)$. Let $\Sigma_{0}$ be a $\sigma$ subalgebra of $\Sigma$. Fix a function $w \in E^{\prime}$. Then $\mathscr{E}\left(w f \mid \Sigma_{0}\right)$ is well defined for all $f \in E$. Now assume in addition that $\mathscr{E}\left(w f \mid \Sigma_{0}\right) \in E$ and put $T f=\mathscr{E}\left(w f \mid \Sigma_{0}\right)$ for all $f \in E$. The linear operator $T: E \rightarrow E$ satisfies the averaging condition. If $w \geq 0$ then $T \geq 0$ and $T$ is order continuous. In the next lemma we collect some other properties of such an operator $T$.

LEMMA 2.1. (i) $T$ can be written as $T=T^{+}-T^{-}$where $T^{+} f=$ $\mathscr{E}\left(w^{+} f \mid \Sigma_{0}\right), T^{-} f=\mathscr{E}\left(w^{-} f \mid \Sigma_{0}\right)$ for all $f \in E$ and $0 \leq T^{+}, T^{-}: E \rightarrow E$. Hence $T$ is order bounded in $E$ and the modulus of $T$ is given by $|T|(f)=T^{+} f+T^{-} f=\mathscr{E}\left(|w| f \mid \Sigma_{0}\right)$ for all $f \in E$.

(ii) $\left\|T f_{n}\right\|_{1} \rightarrow 0$ whenever $f_{n} \rightarrow 0$ a.e. and $\left|f_{n}\right| \leq u \in E$ for all $n$.

(iii) The function $w$ is uniquely determined by $T$.

Proof. (i) It is clearly sufficient to show that $\mathscr{E}\left(w^{+} f \mid \Sigma_{0}\right) \in E$ for all $f \in E$. To this end, write $w^{+}=p w$ for some $p \in L^{\infty}(\Sigma, \mu)$. Then $p f \in E$ and hence $\mathscr{E}\left(w^{+} f \mid \Sigma_{0}\right)=\mathscr{E}\left(w(p f) \mid \Sigma_{0}\right) \in E$ for all $f \in E$. Now it is easily seen that the operator $T_{0}: E \rightarrow E$, defined by $T_{0} f=$ $\mathscr{E}\left(w^{+} f \mid \Sigma_{0}\right)$ for all $f \in E$ is the supremum of $T$ and 0 in the vector lattice of all order bounded linear operators on $E$, i.e. $T_{0}=T^{+}$.

(ii) If $f_{n} \rightarrow 0$ a.e. and $\left|f_{n}\right| \leq u \in E$ for all $n$, then $w f_{n} \rightarrow 0$ a.e. and $\left|w f_{n}\right| \leq|w| u \in L^{1}(\Sigma, \mu)$. By the dominated convergence theorem, $\left\|w f_{n}\right\|_{1} \rightarrow 0$. Since $\mathscr{E}\left(\cdot \mid \Sigma_{0}\right)$ is an $L^{1}$-contraction, it follows that $\left\|T f_{n}\right\|_{1} \rightarrow 0$ as $n \rightarrow \infty$.

(iii) For any $A \in \Sigma$ we have

$$
\int_{A} w d \mu=\int_{\Omega} w \mathbf{1}_{A} d \mu=\int_{\Omega} \mathscr{E}\left(w \mathbf{1}_{A}\right) d \mu=\int_{\Omega} T \mathbf{1}_{A} d \mu,
$$

from which the uniqueness of $w$ follows. 
In connection with (iii) of the above lemma we note that, contrary to the function $w$, the $\sigma$-subalgebra $\Sigma_{0}$ is not uniquely determined by $T$ if $\operatorname{supp}\left(\mathscr{E}\left(w \mid \Sigma_{0}\right) \neq \Omega\right.$.

We end this section with a lemma which will be applied in the sequel. Although the result can be found in [IT], II. 1.2, we conclude the proof for the reader's convenience.

LEMMA 2.2. For a linear subspace $M$ of $L^{\infty}(\Sigma, \mu)$ the following statements are equivalent.

(i) There exists a $\sigma$-subalgebra $\Sigma_{0}$ such that $M=L^{\infty}\left(\Sigma_{0}, \mu\right)$.

(ii) $M$ is a subalgebra of $L^{\infty}(\Sigma, \mu)$ containing the constants such that $f_{n} \in M,\left|f_{n}\right| \leq u \in L^{\infty}(\Sigma, \mu)(n=1,2, \ldots)$ and $f_{n} \rightarrow f$ a.e. imply that $f \in M$.

Proof. Only (ii) $\Rightarrow$ (i) needs a proof. The uniformly closed subalgebra $M$ of $L^{\infty}(\Sigma, \mu)$ contains $\mathbf{1}$, so that (by polynomial approximation) $M$ is a vector sublattice. The collection $\Sigma_{0}=\left\{A \in \Sigma: \mathbf{1}_{A} \in M\right\}$ is a $\sigma$-subalgebra of $\Sigma$. Since any $f \in L^{\infty}\left(\Sigma_{0}, \mu\right)$ is the uniform limit of a sequence of $\Sigma_{0}$-stepfunctions, it follows that $L^{\infty}\left(\Sigma_{0}, \mu\right) \subseteq M$. For the converse inclusion, take $0 \leq f \in M$. For $\alpha \geq 0$ and $n=1,2, \ldots$ we have $n(f-\alpha \mathbf{1})^{+} \wedge \mathbf{1} \in M$. Now $n(f-\alpha \mathbf{1})^{+} \wedge \mathbf{1} \uparrow_{n} \mathbf{1}_{A_{\alpha}}$, where $A_{\alpha}=\{w \in \Omega: f(\omega)>\alpha\}$. Hence $\mathbf{1}_{A_{\alpha}} \in M$, and $M$ is closed for dominated convergence, and so $A_{\alpha} \in \Sigma_{0}$. This shows that $f$ is $\Sigma_{0}$ measurable so that $f \in L^{\infty}\left(\Sigma_{0}, \mu\right)$.

3. The main result. We start this section with an extension of a result due to Shu-Teh Chen Moy ([M, Theorem 2.1]), the proof of which is patterned after [M]. As before, $E$ is an ideal of measurable functions on a finite measure space $(\Omega, \Sigma, \mu)$, satisfying $L^{\infty}(\Sigma, \mu) \subseteq$ $E \subseteq L^{1}(\Sigma, \mu)$.

Proposition 3.1. If $T$ is a linear operator on $E$ for which

(i) $T f \in L^{\infty}(\Sigma, \mu)$ whenever $f \in L^{\infty}(\Sigma, \mu)$,

(ii) $\left\|T f_{n}\right\|_{1} \rightarrow 0$ for all sequences $\left\{f_{n}\right\}_{n=1}^{\infty}$ in $E$ such that $\left|f_{n}\right| \leq u$ $(n=1,2, \ldots)$ for some $u \in E$ and $f_{n} \rightarrow 0$ a.e.,

(iii) $T(f \cdot T g)=T f \cdot T g$ for all $f \in L^{\infty}(\Sigma, \mu)$ and all $g \in E$, then there exists a $\sigma$-subalgebra $\Sigma_{0}$ of $\Sigma$ and there exists $w \in E^{\prime}$ such that $T f=\mathscr{E}\left(w f \mid \Sigma_{0}\right)$ for all $f \in E$. 
Proof. The subalgebra

$$
\mathscr{A}=\left\{h \in L^{\infty}(\Sigma, \mu): T(f h)=T f \cdot h \text { for all } f \in L^{\infty}(\Sigma, \mu)\right\}
$$

of $L^{\infty}(\Sigma, \mu)$ contains 1 and, by (ii), is closed under dominated a.e. convergence in $L^{\infty}(\Sigma, \mu)$. Therefore, by Lemma 2.2, there exists a $\sigma$ subalgebra $\Sigma_{0}$ of $\Sigma$ such that $\mathscr{A}=L^{\infty}\left(\Sigma_{0}, \mu\right)$. It follows from condition (iii) that $T\left(L^{\infty}(\Sigma, \mu)\right) \subseteq \mathscr{A}$.

We now show that $T f$ if $\Sigma_{0}$-measurable for all $f \in E$. Given $f \in E$ there exist $f_{n} \in L^{\infty}(\Sigma, \mu)(n=1,2, \ldots)$ such that $\left|f_{n}\right| \leq|f|$ and $f_{n} \rightarrow f$ a.e. As observed above, $T f_{n} \in L^{\infty}\left(\Sigma_{0}, \mu\right)$ for all $n$, and by (ii), $T f_{n} \rightarrow$ $T f$ in $L^{1}$-norm, so that $T f$ is $\Sigma_{0}$-measurable. In order to obtain the function $w$ we define the set function $\nu$ on $\Sigma$ by

$$
\nu(A)=\int_{\Omega} T \mathbf{1}_{A} d \mu
$$

for all $A \in \Sigma$, which is easily seen to be $\sigma$-additive by (ii). The signed measure $\nu$ is absolutely continuous with respect to $\mu$ and the RadonNikodym derivative of $\nu$ with respect to $\mu$ is denoted by $w$. We claim that $w \in E^{\prime}$ and that

$$
\int_{\Omega} T f d \mu=\int_{\Omega} w f d \mu, \quad f \in E .
$$

Indeed, take $0 \leq f \in E$ and let $\left\{t_{n}\right\}_{n=1}^{\infty}$ be a sequence of $\Sigma$-stepfunctions such that $0 \leq t_{n} \uparrow f$ a.e.. Let $A=\{\omega \in \Omega: w(\omega)>0\}$ and $B=\Omega \backslash A$. It follows from the definition of $w$ that

$$
\int_{\Omega} T\left(\mathbf{1}_{A} t_{n}\right) d \mu=\int_{\Omega}\left(\mathbf{1}_{A} t_{n}\right) w d \mu=\int_{\Omega} w^{+} t_{n} d \mu
$$

for all $n$. Since $0 \leq \mathbf{1}_{A} t_{n} \uparrow \mathbf{1}_{A} f$ a.e. condition (ii) implies that $T\left(\mathbf{1}_{A} t_{n}\right) \rightarrow T\left(\mathbf{1}_{A} f\right)$ in $L^{1}$-norm, so $\int_{\Omega} T\left(\mathbf{1}_{A} t_{n}\right) d \mu \rightarrow \int_{\Omega} T\left(\mathbf{1}_{A} f\right) d \mu$. On the other hand, $\int_{\Omega} w^{+} t_{n} f d \mu \uparrow \int_{\Omega} w^{+} f d \mu$; hence

$$
\int_{\Omega} w^{+} f d \mu=\int_{\Omega} T\left(\mathbf{1}_{A} f\right) d \mu<\infty .
$$

Similarly we find that

$$
\int_{\Omega} w^{-} f d \mu=-\int_{\Omega} T\left(\mathbf{1}_{B} f\right) d \mu<\infty
$$

and the claim is proved.

To finish the proof, observe first that (once more by (ii)), $T(f h)=$ $T f \cdot h$ for all $f \in E$ and $h \in L^{\infty}\left(\Sigma_{0}, \mu\right)$. Now take $D \in \Sigma_{0}$. Then we 
have for all $f \in E$,

$$
\begin{aligned}
\int_{D} T f d \mu & =\int_{\Omega} T f \cdot \mathbf{1}_{D} d \mu=\int_{\Omega} T\left(f \mathbf{1}_{D}\right) d \mu=\int_{\Omega} w f \mathbf{1}_{D} d \mu \\
& =\int_{D} w f d \mu .
\end{aligned}
$$

Since $T f$ is $\Sigma_{0}$-measurable we may conclude that $T f=\mathscr{E}\left(w f \mid \Sigma_{0}\right)$ for all $f \in E$.

REMARKS. 3.2(i) For positive operators $T$, it is evident that the above condition (ii) is equivalent to the order continuity of $T$ (i.e. $f_{n} \downarrow 0$ a.e. in $E$ implies that $T f_{n} \downarrow 0$ a.e.). Furthermore, if $E$ is a Banach function space with order continuous norm $\|\cdot\|_{E}$, then (ii) is satisfied for any norm bounded operator $T$ on $E$. Indeed, if $\left|f_{n}\right| \leq$ $u \in E$ and $f_{n} \rightarrow 0$ a.e., then the elements $v_{n}=\sup _{k \geq n}\left|f_{k}\right|$ satisfy $v_{n} \downarrow 0$ a.e., so $\left\|v_{n}\right\|_{E} \downarrow 0$, which implies $\left\|f_{n}\right\|_{E} \rightarrow 0$; hence $\left\|T f_{n}\right\|_{E} \rightarrow 0$. Consequently $\left\|T f_{n}\right\|_{1} \rightarrow 0$, since $\|\cdot\|_{1} \leq C\|\cdot\|_{E}$ for some constant $C>0$. This observation shows that the above proposition is an exact generalization of Moy's result on $L^{p}$-spaces [M].

(ii) Suppose that $T: E \rightarrow E$ is given by $T f=\mathscr{E}\left(w f \mid \Sigma_{0}\right), f \in E$, for some $\sigma$-subalgebra $\Sigma_{0}$ of $\Sigma$ and some $w \in E^{\prime}$. In general, such a $T$ need not be a projection. In fact, $T^{2}=T$ if and only $\mathscr{E}\left(w \mid \Sigma_{0}\right)=\mathbf{1}_{S}$ and $\operatorname{supp}(w) \subseteq S$ for some $S \in \Sigma_{0}$. Indeed, if $T^{2}=T$, then $T \mathbf{1}=$ $T(\mathbf{1} \cdot T \mathbf{1})=(T \mathbf{1})^{2}$, so $\mathscr{E}\left(w \mid \Sigma_{0}\right)=T \mathbf{1}=\mathbf{1}_{S}$ for some $S \in \Sigma_{0}$. Note that $T f=T(\mathbf{1} \cdot T f)=T \mathbf{1} \cdot T f$, so $T f=\mathbf{1}_{S} T f$ for all $f \in E$. Writing $|w|=w p$ with $p \in L^{\infty}(\Sigma, \mu)$ we get

$$
\int_{\Omega \backslash S}|w| d \mu=\int_{\Omega \backslash S} \mathscr{E}\left(w p \mid \Sigma_{0}\right) d \mu=\int_{\Omega \backslash S} T p d \mu=\int_{\Omega \backslash S} \mathbf{1}_{S} T p d \mu=0 ;
$$

hence $\operatorname{supp}(w) \subseteq S$. Conversely, if $\mathscr{E}\left(w \mid \Sigma_{0}\right)=\mathbf{1}_{S}$ and $\operatorname{supp}(w) \subseteq S$ then

$$
\begin{aligned}
\int_{\Omega \backslash S}|T f| d \mu & =\int_{\Omega \backslash S}\left|\mathscr{E}\left(w f \mid \Sigma_{0}\right)\right| d \mu \leq \int_{\Omega \backslash S} \mathscr{E}\left(|w f| \mid \Sigma_{0}\right) d \mu \\
& =\int_{\Omega \backslash S}|w f| d \mu=0 .
\end{aligned}
$$

which implies that $\operatorname{supp}(T f) \subseteq S$ for all $f \in E$. Hence $T f=\mathbf{1}_{S} T f$ and consequently $T^{2} f=T(\mathbf{1} \cdot T f)=T \mathbf{1} \cdot T f=\mathbf{1}_{S} T f=T f$ for all $f \in E$.

The condition that $\operatorname{supp}(w) \subseteq S$ in the above equivalence cannot be omitted, as is illustrated in the following example. Let $E=L^{1}[0,1]$ 
(with respect to Lebesgue measure) and let $\Sigma_{0}$ be the $\sigma$-subalgebra generated by $\left[0, \frac{1}{2}\right]$. The function $w=\mathbf{1}_{[0,3 / 4]}-\mathbf{1}_{(3 / 4,1]}$ satisfies $\mathscr{E}\left(w \mid \Sigma_{0}\right)=$ $\mathbf{1}_{[0,1 / 2]}$ but $\operatorname{supp}(w)$ is not contained in $\left[0, \frac{1}{2}\right]$.

If $w \geq 0$, which is obviously equivalent to $T \geq 0$, then $\mathscr{E}\left(w \mid \Sigma_{0}\right)=$ $\mathbf{1}_{S}$ does imply that $\operatorname{supp}(w) \subseteq S$, as $\int_{\Omega \backslash S} w d \mu=\int_{\Omega \backslash S} \mathscr{E}\left(w \mid \Sigma_{0}\right) d \mu=$ $\int_{\Omega \backslash S} \mathbf{1}_{S} d \mu=0$.

Note in this connection that an averaging projection need not be positive, which is shown by taking $E=L^{1}[0,1], \Sigma_{0}=\{\phi,[0,1]\}$ and $w=41_{[0,1 / 2]}-21_{(1 / 2,1]}$.

Finally recall that a positive function $w$ is called a weight function with respect to the $\sigma$-subalgebra $\Sigma_{0}$ if $\mathscr{E}\left(w \mid \Sigma_{0}\right)=\mathbf{1}_{S}$, where $S=\operatorname{supp}(w)$ (see [D1]). Clearly, if $w$ is a weight function for $\Sigma_{0}$, then the corresponding operator $T$ is a positive projection. The converse, however, is not true. By way of example, take $E=L^{1}[0,1]$, $\Sigma_{0}=\{\phi,[0,1]\}$ and $w=2 \mathbf{1}_{\left[0, \frac{1}{2}\right]}$.

Next we will use the above proposition to describe a certain class of positive projections.

Proposition 3.3. For a linear operator $T: E \rightarrow E$ the following statements are equivalent.

(i) $T$ is positive and order continuous, $T^{2}=T, T \mathbf{1}=\mathbf{1}$ and the range $R(T)$ of $T$ is a sublattice.

(ii) There exist a $\sigma$-subalgebra $\Sigma_{0}$ of $\Sigma$ and a function $0 \leq w \in E^{\prime}$ with $\mathscr{E}\left(w \mid \Sigma_{0}\right)=\mathbf{1}$ such that $T f=\mathscr{E}\left(w f \mid \Sigma_{0}\right)$ for all $f \in E$.

Proof. (i) $\Rightarrow$ (ii) First note that $T \geq 0$ and $T \mathbf{1}=\mathbf{1}$ imply that $T f \in L^{\infty}(\Sigma, \mu)$ whenever $f \in L^{\infty}(\Sigma, \mu)$. Denote by $T_{\infty}$ the restriction of $T$ to $L^{\infty}(\Sigma, \mu)$. Since $T$ is a projection, $R\left(T_{\infty}\right)=R(T) \cap L^{\infty}(\Sigma, \mu)$, so $R\left(T_{\infty}\right)$ is a sublattice of $L^{\infty}(\Sigma, \mu)$. Moreover, since $T_{\infty}$ is positive, $T_{\infty}$ is $L^{\infty}$-continuous so $R\left(T_{\infty}\right)$ is closed in $L^{\infty}(\Sigma, \mu)$. Hence, $R\left(T_{\infty}\right)$ is a uniformly closed vector sublattice of $L^{\infty}(\Sigma, \mu)$ containing 1 , and so $R\left(T_{\infty}\right)$ is a subalgebra (see [D2] or [HP1], Theorem 3.5). Now by a result of J. L. Kelley ([K], Theorem 2.5; see also [HP2], Theorem 6.1) it follows that $T_{\infty}$ is an averaging operator in $L^{\infty}(\Sigma, \mu)$ so that, by the order continuity of $T$, we have $T(f T g)=T f \cdot T g$ for all $f \in L^{\infty}(\Sigma, \mu)$ and $g \in E$. As observed in Remark 3.2(i), if $T$ is positive and order continuous, then $T$ satisfies also condition (ii) of Proposition 3.1, and hence this proposition yields the desired result. 
(ii) $\Rightarrow$ (i) Given $f \in R(T)$ we have $f=\mathscr{E}\left(w f \mid \Sigma_{0}\right)$ and we can write $|f|=p f$ for some $p \in L^{\infty}\left(\Sigma_{0}, \mu\right)$. Hence $|f|=p \mathscr{E}\left(w f \mid \Sigma_{0}\right)=$ $\mathscr{E}\left(w p f \mid \Sigma_{0}\right)=\mathscr{E}\left(w|f| \mid \Sigma_{0}\right)$, showing that $|f| \in R(T)$, so $R(T)$ is a sublattice. The remaining statements concerning $T$ are evident.

In general, the range of a positive order continuous projection $T$ for which $T 1=1$ need not be a sublattice. This is shown by the projection $T$ in $\mathbf{R}^{3}$ (with coordinatewise ordering) onto the plane $x_{1}+x_{2}-2 x_{3}=0$ (with respect to the standard basis) along the direction of the third basis vector.

As an immediate consequence of the above proposition we obtain the following result.

COROLlaRY 3.4. For a linear operator $T: E \rightarrow E$ the following statements are equivalent.

(i) $T$ is a strictly positive (i.e. $T f>0$ if $f>0$ ) order continuous projection with $T \mathbf{1}=\mathbf{1}$.

(ii) There exist a $\sigma$-subalgebra $\Sigma_{0}$ of $\Sigma$ and a strictly positive function $w \in E^{\prime}$ with $\mathscr{E}\left(w \mid \Sigma_{0}\right)=\mathbf{1}$ such that $T f=\mathscr{E}\left(w f \mid \Sigma_{0}\right)$ for all $f \in E$.

Proof. By Proposition 3.3, we only have to observe that the range of a strictly positive projection is a sublattice. In fact, if $T f=f$, then $f^{+}=(T f)^{+} \leq T\left(f^{+}\right)$and $T\left(T\left(f^{+}\right)-f^{+}\right)=0$ so $T\left(f^{+}\right)=f^{+}$.

REMARK 3.5. If $w$ is a strictly positive function in $E^{\prime}$, then the measure $\nu$ defined by $d \nu=w d \mu$ is equivalent with $\mu$ and $E \subseteq L^{1}(\Sigma, \nu)$. Then $\mathscr{E}^{\mu}\left(w \mid \Sigma_{0}\right)$ is strictly positive and

$$
\mathscr{E}^{\nu}\left(f \mid \Sigma_{0}\right)=\frac{\mathscr{E}^{\mu}\left(w f \mid \Sigma_{0}\right)}{\mathscr{E}^{\mu}\left(w \mid \Sigma_{0}\right)}
$$

for all $f \in L^{1}(\Sigma, \nu)$. In particular, if $w$ is a weight function for $\Sigma_{0}$, then $\mathscr{E}^{\nu}\left(f \mid \Sigma_{0}\right)=\mathscr{E}^{\mu}\left(w f \mid \Sigma_{0}\right)$ for all $f \in L^{1}(\Sigma, \mu)$. Therefore, the result of the above corollary can be reformulated as follows: the linear operator $T$ from $E$ into itself is a strictly positive order continuous projection with $T 1=1$ if and only if there exists a $\sigma$-subalgebra $\Sigma_{0}$ of $\Sigma$ and an equivalent measure $\nu$ on $\Sigma$ such that $E \subseteq L^{1}(\Sigma, \nu)$ and $T f=\mathscr{E}^{\nu}\left(f \mid \Sigma_{0}\right)$ for all $f \in E$. This is the main result of [dJ].

Let $(\Omega, \Sigma, \mu)$ and $E$ be as above. In the following proposition we shall relax the condition that $T \mathbf{1}=\mathbf{1}$ and we shall assume instead that $T 1$ is strictly positive. 
Proposition 3.6. For a linear operator $T: E \rightarrow E$ the following statements are equivalent.

(i) $T$ is a positive order continuous projection onto a sublattice such that $T \mathbf{1}$ is strictly positive.

(ii) There exist a $\sigma$-subalgebra $\Sigma_{0}$ of $\Sigma, 0 \leq w \in E^{\prime}$ and a strictly positive function $k \in L^{1}(\Sigma, \mu)$ with $\mathscr{E}\left(w k \mid \Sigma_{0}\right)=1$, such that $T f=$ $k \mathscr{E}\left(w f \mid \Sigma_{0}\right)$ for all $f \in E$. Moreover, if we choose $k$ such that $\mathscr{E}\left(k \mid \Sigma_{0}\right)=$ 1 then both $w$ and $k$ are uniquely determined by $T$.

Proof. (i) $\Rightarrow$ (ii) The measure $\nu$ on $\Sigma$, defined by $d \nu=T \mathbf{1} d \mu$ is equivalent to $\mu$, so $L^{0}(\Sigma, \nu)=L^{0}(\Sigma, \mu)$. The set $F=\left\{(T \mathbf{1})^{-1} f: f \in\right.$ $E\}$ is an ideal of measurable functions in $L^{1}(\Sigma, \nu)$ and the mapping $M: E \rightarrow F$, defined by $M f=(T \mathbf{1})^{-1} f$ for all $f \in E$ is a lattice isomorphism from $E$ onto $F$. Furthermore, $L^{\infty}(\Sigma, \nu) \subset F$, as $M(T \mathbf{1})=\mathbf{1} \in F$. The mapping $S=M T M^{-1}$ is a positive projection in $F$ and $S \mathbf{1}=\mathbf{1}$. The range $R(S)=M[R(T)]$ is a sublattice of $F$. As before, we denote by $E^{\prime}$ the associate space of $E$ with respect to $\mu$, whereas by $F^{\prime}$ we denote momentarily the associate space of $F$ with respect to $\nu$. It is easily verified that $F^{\prime}=E^{\prime}$.

It follows from Proposition 3.3 that there exists a $\sigma$-subalgebra $\Sigma_{0}$ of $\Sigma$ and function $0 \leq w \in F^{\prime}$ such that $S g=\mathscr{E}^{\nu}\left(w g \mid \Sigma_{0}\right)$ for all $g \in F$, and hence

$$
T f=T \mathbf{1} \cdot \mathscr{E}^{\nu}\left(w(T \mathbf{1})^{-1} f \mid \Sigma_{0}\right)
$$

for all $f \in E$. Using the formula in Remark 3.5 we find that

$$
\mathscr{E}^{\nu}\left(w(T \mathbf{1})^{-1} f \mid \Sigma_{0}\right)=\frac{\mathscr{E}^{\mu}\left(w f \mid \Sigma_{0}\right)}{\mathscr{E}^{\mu}\left(T \mathbf{1} \mid \Sigma_{0}\right)}
$$

for all $f \in E$, and so

$$
T f=\frac{T \mathbf{1}}{\mathscr{E} \mu\left(T \mathbf{1} \mid \Sigma_{0}\right)} \mathscr{E}^{\mu}\left(w f \mid \Sigma_{0}\right)
$$

for all $f \in E$. The function $k=T \mathbf{1} \cdot\left[\mathscr{E}^{\mu}\left(T \mathbf{1} \mid \Sigma_{0}\right)\right]^{-1}$ satisfies $0 \leq k \in$ $L^{1}(\Sigma, \mu), \mathscr{E}^{\mu}\left(k \mid \Sigma_{0}\right)=\mathbf{1}$, and is strictly positive. Since

$$
\begin{aligned}
T \mathbf{1} & =T^{2} \mathbf{1}=k \mathscr{E}^{\mu}\left(w k \mathscr{E}^{\mu}\left(w \mid \Sigma_{0}\right) \mid \Sigma_{0}\right) \\
& =k \mathscr{E}^{\mu}\left(w \mid \Sigma_{0}\right) \mathscr{E}^{\mu}\left(w k \mid \Sigma_{0}\right)=T \mathbf{1} \mathscr{E}^{\mu}\left(w k \mid \Sigma_{0}\right),
\end{aligned}
$$


we find that $\mathscr{E}^{\mu}\left(w k \mid \Sigma_{0}\right)=1$. Moreover, it follows from $\mathscr{E}^{\mu}\left(k \mid \Sigma_{0}\right)=1$ that for all $A \in \Sigma$ we have

$$
\begin{aligned}
\int_{\Omega} T \mathbf{1}_{A} d \mu & =\int_{\Omega} k \mathscr{E}^{\mu}\left(w \mathbf{1}_{A} \mid \Sigma_{0}\right) d \mu \\
& =\int_{\Omega} \mathscr{E}^{\mu}\left(k \mathscr{E}^{\mu}\left(w \mathbf{1}_{A} \mid \Sigma_{0}\right) \mid \Sigma_{0}\right) d \mu \\
& =\int_{\Omega} \mathscr{E}^{\mu}\left(w \mathbf{1}_{A} \mid \Sigma_{0}\right) \mathscr{E}^{\mu}\left(k \mid \Sigma_{0}\right) d \mu \\
& =\int_{\Omega} \mathscr{E}^{\mu}\left(w \mathbf{1}_{A} \mid \Sigma_{0}\right) d \mu=\int_{A} w d \mu,
\end{aligned}
$$

which shows that $w$ is uniquely determined by $T$. The uniqueness of $k$ is now immediate from $T \mathbf{1}=k \mathscr{E}^{\mu}\left(w \mid \Sigma_{0}\right)$.

(ii) $\Rightarrow$ (i). Now suppose that $T: E \rightarrow E$ is given by $T f=k \mathscr{E}\left(w f \mid \Sigma_{0}\right)$ for all $f \in E$, where $\Sigma_{0}$ is a $\sigma$-subalgebra of $\Sigma, k$ is a strictly positive function in $L^{1}(\Sigma, \mu), 0 \leq w \in E^{\prime}$ and $\mathscr{E}\left(w k \mid \Sigma_{0}\right)=1$. Obviously, $T$ is positive and order continuous. In order to show that $T 1$ is strictly positive, it suffices to verify that $\mathscr{E}\left(w \mid \Sigma_{0}\right)$ is strictly positive. To this end, put $A=\left\{\omega \in \Omega: \mathscr{E}\left(w \mid \Sigma_{0}\right)(\omega)=0\right\}$. Then $\int_{A} w d \mu=\int_{A} \mathscr{E}\left(w \mid \Sigma_{0}\right) d \mu=0$, so $w$ is zero on $A$, which implies that

$$
\mu(A)=\int_{A} \mathscr{E}\left(w k \mid \Sigma_{0}\right) d \mu=\int_{A} w k d \mu=0 .
$$

It is an immediate consequence of $\mathscr{E}\left(w k \mid \Sigma_{0}\right)=\mathbf{1}$ that $T^{2}=T$. Finally we prove that $R(T)$ is a sublattice of $E$. If $f \in R(T)$, then $f=$ $k \mathscr{E}\left(w f \mid \Sigma_{0}\right)$ and hence $|f|=k\left|\mathscr{E}\left(w f \mid \Sigma_{0}\right)\right|=p k \mathscr{E}\left(w f \mid \Sigma_{0}\right)$, for some $p \in L^{\infty}\left(\Sigma_{0}, \mu\right)$, so that $|f|=k \mathscr{E}\left(w p f \mid \Sigma_{0}\right)=T(p f)$.

The following corollary is now obvious.

CoRollary 3.7. For a linear operator $T: E \rightarrow E$ the following statements are equivalent.

(i) $T$ is a strictly positive order continuous projection such that T1 is strictly positive.

(ii) There exist a $\sigma$-subalgebra $\Sigma_{0}$ of $\Sigma$ and strictly positive functions $w$ in $E^{\prime}$ and $k$ in $L^{1}(\Sigma, \mu)$ with $\mathscr{E}\left(w k \mid \Sigma_{0}\right)=1$ such that $T f=$ $k \mathscr{E}\left(w f \mid \Sigma_{0}\right)$ for all $f \in E$.

The next result extends Proposition 3.6. The proof bears resemblance to the proof of Proposition 2.2 in [D1]. 
Proposition 3.8. If $T: E \rightarrow E$ is a linear operator, then the statements (i) and (ii) are equivalent.

(i) $T$ is a positive order continuous projection onto a sublattice.

(ii) $T$ can be written uniquely in the form $T=T_{1}+T_{2}$, where

(a) $T_{1}$ is given by

$$
T_{1} f=k \mathscr{E}\left(w f \mid \Sigma_{0}\right), \quad f \in E,
$$

where $\Sigma_{0}$ is a $\sigma$-subalgebra of $\Sigma, 0 \leq k \in L^{1}(\Sigma, \mu), 0 \leq w \in E^{\prime}$, $\mathscr{E}\left(w k \mid \Sigma_{0}\right)=\mathbf{1}_{\operatorname{supp}(k)}$, and $\operatorname{supp}(w) \subseteq \operatorname{supp}(k)$. Moreover, if we choose $k$ such that $\mathscr{E}\left(k \mid \Sigma_{0}\right)=\mathbf{1}_{\text {supp }(k)}$, then $w$ and $k$ are unique.

(b) $T_{2}$ is a positive order continuous linear operator on $E$ such that $T_{1} T_{2}=T_{2}$ and $T_{2} T_{1}=0$ (and hence $\left.T_{2}^{2}=0\right)$.

Proof (i) $\Rightarrow$ (ii). To show the uniqueness of the decomposition, it suffices to show that $T_{1} f=T\left(f \mathbf{1}_{S}\right)$ where $S=\operatorname{supp}(T(\mathbf{1}))$. We observe first that $\operatorname{supp}(k)=\operatorname{supp}\left(\mathscr{E}\left(w \mid \Sigma_{0}\right)\right)$. Since $\operatorname{supp}(w) \subseteq \operatorname{supp}(k)$ and $\operatorname{supp}(k) \in \Sigma_{0}$, it is clear that $\operatorname{supp}\left(\mathscr{E}\left(w \mid \Sigma_{0}\right)\right) \subseteq \operatorname{supp}(k)$. If we set

$$
A=\left\{\omega \in \operatorname{supp}(k): \mathscr{E}\left(w \mid \Sigma_{0}\right)(\omega)=0\right\}
$$

then it follows as in the proof of the implication (ii) $\Rightarrow$ (i) of Proposition 3.6 above that $\mu(A)=0$ and so $\operatorname{supp}\left(\mathscr{E}\left(w \mid \Sigma_{0}\right)\right)=\operatorname{supp}(k)$. This shows that $\operatorname{supp}\left(T_{1}(\mathbf{1})\right)=\operatorname{supp}(k)$ and from this it follows that $\operatorname{supp}(k)=\operatorname{supp}(T(\mathbf{1}))=S$. In fact, from $0 \leq T_{1}(\mathbf{1}) \leq T(\mathbf{1})$, it follows that $\operatorname{supp}(k) \subseteq \operatorname{supp}(T(\mathbf{1}))$. On the other hand,

$$
T(\mathbf{1})=T_{1}(\mathbf{1})+T_{2}(\mathbf{1})=T_{1}\left(\mathbf{1}+T_{2}(\mathbf{1})\right)
$$

implies that $\operatorname{supp} T(\mathbf{1}) \subseteq \operatorname{supp}(k)$ and so equality holds. If now $0 \leq$ $f \in E$, we have that $n T_{1}(\mathbf{1}) \wedge f \mathbf{1}_{S} \uparrow f \mathbf{1}_{S}$ and so, by order continuity of $T_{2}$, it follows that $T_{2}\left(n T_{1}(\mathbf{1}) \wedge f \mathbf{1}_{S}\right) \uparrow T_{2}\left(f \mathbf{1}_{S}\right)$. On the other hand

$$
0 \leq T_{2}\left(n T_{1}(\mathbf{1}) \wedge f \mathbf{1}_{S}\right) \leq n T_{2} T_{1} \mathbf{1}=0,
$$

so that $T_{2}\left(f \mathbf{1}_{S}\right)=0$. Further $T_{1}\left(f \mathbf{1}_{\Omega \backslash S}\right)=0$ for all $f \in E$ since $\operatorname{supp}(w) \subseteq S$ and this implies that $T_{1} f=T_{1}\left(f \mathbf{1}_{S}\right)$ for all $f \in E$. Hence

$$
T_{1} f=T_{1}\left(f \mathbf{1}_{S}\right)=T_{1}\left(f \mathbf{1}_{S}\right)+T_{2}\left(f \mathbf{1}_{S}\right)=T\left(f \mathbf{1}_{S}\right)
$$

and so the decomposition is unique.

Next we show the existence of such a decomposition. Let $S=$ $\operatorname{supp}(T \mathbf{1})$. Since $T(f \wedge n \mathbf{1}) \uparrow T f$ for all $0 \leq f \in E$, and $0 \leq T(f \wedge n \mathbf{1}) \leq$ $n T \mathbf{1}$ for all $n$, it follows that $\operatorname{supp}(T f) \subseteq S$, and so $T f=\mathbf{1}_{S} T f$ for all 
$f \in E$. Now define $T_{1} f=T\left(f \mathbf{1}_{S}\right)$ for all $f \in E$ and set $T_{2}=T-T_{1}$. Obviously $T_{1}$ and $T_{2}$ are positive and order continuous, and $T_{1}^{2} f=$ $T\left(T\left(f \mathbf{1}_{S}\right) \mathbf{1}_{S}\right)=T^{2}\left(f \mathbf{1}_{S}\right)=T_{1} f$ for all $f \in E$. The relations $T_{1} T_{2}=T_{2}$ and $T_{2} T_{1}=0$ are easily verified. An application of Proposition 3.6 to the restriction of $T_{1}$ to the ideal $E_{1}=\{f \mid S: f \in E\}$ of measurable functions on $S$ yields the desired $\sigma$-subalgebra $\Sigma_{0}$ and the functions $k$ and $w$. We leave the straightforward details to the reader.

(ii) $\Rightarrow$ (i). If $T=T_{1}+T_{2}$ with $T_{1}$ and $T_{2}$ as in (a) and (b), then $\mathscr{E}\left(w k \mid \Sigma_{0}\right)=\mathbf{1}_{\text {supp }(k)}$ implies that $T_{1}^{2}=T_{1}$. It now follows easily that $T^{2}=T$. Furthermore, $R\left(T_{1}\right)=R(T)$. Indeed, if $f \in R(T)$, then $T_{1} f=T_{1}\left(T_{1} f+T_{2} f\right)=T_{1} f+T_{2} f=T f=f$, so $R(T) \subseteq R\left(T_{1}\right)$. Conversely, if $f \in R\left(T_{1}\right)$ then $T f=T_{1}\left(T_{1} f\right)+T_{2}\left(T_{1} f\right)=T_{1} f=f$, which shows that $R\left(T_{1}\right) \subseteq R(T)$. It follows from Proposition 3.6 that $R(T)=R\left(T_{1}\right)$ is a sublattice of $E$. The remaining statements on $T$ are evident.

It should be observed that $T_{2}=0$ in the above decomposition if and only if $T f=0$ for all $f \in R(T)^{d}$. The latter condition is the regularity condition $(*)$ of Douglas ([D1], $\S 1)$.

COROLlary 3.9. For a linear operator $T: E \rightarrow E$ the following statements are equivalent.

(i) $T$ is a strictly positive order continuous projection.

(ii) $T$ can be written uniquely in the form $T=T_{1}+T_{2}$, where

(a) $T_{1}$ is given by

$$
T_{1} f=k \mathscr{E}\left(w f \mid \Sigma_{0}\right), \quad f \in E
$$

where $\Sigma_{0}$ is a $\sigma$-subalgebra of $\Sigma, 0 \leq k \in L^{1}(\Sigma, \mu), 0 \leq w \in E^{\prime}$, $\mathscr{E}\left(k \mid \Sigma_{0}\right)=\mathscr{E}\left(w k \mid \Sigma_{0}\right)=\mathbf{1}_{\operatorname{supp}(k)}$ and $\operatorname{supp}(w)=\operatorname{supp}(k)$;

(b) $T_{2}$ is a positive order continuous linear operator on $E$, which is strictly positive on $\left\{f \mathbf{1}_{\Omega \backslash \operatorname{supp}(k)}: f \in E\right\}$, such that $T_{1} T_{2}=T_{2}$ and $T_{2} T_{1}=0$.

Proof. (ii) $\Rightarrow$ (i). It follows from (a) that $T_{1}$ is strictly positive on the ideal $\left\{f \mathbf{1}_{\operatorname{supp}(k)}: f \in E\right\}$ and hence $T$ is strictly positive on $E$.

(i) $\Rightarrow$ (ii). As observed before, the strict positivity of $T$ implies that $R(T)$ is a sublattice of $E$, so we can apply Proposition 3.8. Therefore, it remains to show that $\operatorname{supp}(w)=\operatorname{supp}(k)$ and that $T_{2}$ is strictly positive on $\left.\left\{f \mathbf{1}_{\Omega \backslash \operatorname{supp}(k)}\right): f \in E\right\}$. This follows, however, immediately 
from the fact that $T_{1} f=T\left(f \mathbf{1}_{\operatorname{supp}(k)}\right)$ and $T_{2} f=T\left(f \mathbf{1}_{\Omega \backslash \operatorname{supp}(k)}\right)$ for all $f \in E$.

Now we are in a position to prove the main result of this paper. This result was proved by different methods by V. G. Kulakova ([Ku], Theorem 2) for the special class of weakly sequentially complete rearrangement invariant Banach function spaces.

THEOREM 3.10. Let $0 \leq T: E \rightarrow E$ be an order continuous projection. Then $\Omega$ can be written as a disjoint union $\Omega=S_{1} \cup S_{2} \cup S_{3}\left(S_{i} \in \Sigma\right)$ such that with respect to the corresponding direct sum decomposition $E=E_{1} \oplus E_{2} \oplus E_{3}$, with $E_{i}=\left\{f \mathbf{1}_{S_{1}}: f \in E\right\}$ the operator $T$ has the matrix representation

$$
\left[\begin{array}{ccc}
T_{11} & T_{12} & 0 \\
0 & 0 & 0 \\
T_{31} & T_{32} & 0
\end{array}\right],
$$

where $0 \leq T_{i j}: E_{j} \rightarrow E_{i}$ are order continuous, $T_{11}$ and $T_{12}$ are strictly positive and

$$
T_{11}^{2}=T_{11}, \quad T_{11} T_{12}=T_{12}, \quad T_{31} T_{11}=T_{31}, \quad T_{31} T_{12}=T_{32} .
$$

Furthermore, there exists a $\sigma$-subalgebra $\Sigma_{0}$ of $\Sigma$ and there exist functions $0 \leq w \subset E^{\prime}, 0 \leq k \in L^{1}(\Sigma, \mu)$ such that $T_{11} f=k \mathscr{E}\left(w f \mid \Sigma_{0}\right)$ for all $f \in E_{1}, \mathscr{E}\left(k \mid \Sigma_{0}\right)=\mathscr{E}\left(w k \mid \Sigma_{0}\right)=\mathbf{1}_{S_{1}}$ and $\operatorname{supp}(k)=\operatorname{supp}(w)=S_{1}$. This decomposition is uniquely determined by $T$.

Moreover, any operator of this form is a positive projection in $E$.

Proof. Since $T$ is order continuous, the ideal $E_{3}=\{f \in E: T|f|=$ $0\}$ is closed with respect to monotone convergence in $E$, and so $E_{3}=$ $\left\{f \mathbf{1}_{S_{3}}: f \in E\right\}$ for some $S_{3} \in \Sigma$. Define the projection $P$ in $E$ by $P f=f 1_{\Omega \backslash S_{3}}$ for all $f \in E$. Then $T P=T$, so $(P T)^{2}=P T$ and hence $P T$ is a positive projection in $E$. Let $\tilde{T}: E_{3}^{d} \rightarrow E_{3}^{d}$ be the restriction of $P T$ to $E_{3}^{d}$. It follows from $T=T^{2}=T P T$ that $\tilde{T}$ is strictly positive, so $R(\tilde{T})$ is a sublattice of $E_{3}^{d}$. Take $E_{1}=R(\tilde{T})^{d d} \subseteq E_{3}^{d}$ and $E_{2}=R(\tilde{T})^{d} \cap E_{3}^{d}$. Then $E_{i}=\left\{f 1_{S_{i}}: f \in E\right\}$ for some $S_{i} \in \Sigma$ $(i=1,2)$. An application of Corollary 3.9 to the operator $\tilde{T}$ yields the strictly positive operators $T_{11}$ and $T_{12}$. The verification of the remaining statements is straightforward and is therefore left to the reader. 
4. Averaging contractions and contractive projections. Throughout this section, we assume that $\left(E,\|\cdot\|_{E}\right)$ is a Banach function space on some finite measure space $(\Omega, \Sigma, \mu)$ for which $L^{\infty}(\Sigma, \mu) \subseteq E \subseteq$ $L^{1}(\Sigma, \mu)$. The linear map $T: E \rightarrow E$ is called averaging if $T(f T g)=$ $T f \cdot T g$ for all $f \in L^{\infty}(\Sigma, \mu)$ and all $g \in E$. It has been shown by Rota ([Ro], Theorem 1) that if $(\Omega, \Sigma, \mu)$ is a probability space, then each averaging contraction on $L^{p}(\Sigma, \mu), 1 \leq p<\infty$, which preserves 1 is a conditional expectation operator. We now indicate how the results of the previous section yield extensions of Rota's theorem to the setting of Banach function spaces.

We start with a simple observation, which in the case that $E$ is an $L^{p}$-space $(1 \leq p<\infty)$, may be found in [Ro], Proposition 2 .

LEMMA 4.1. If the contraction $T: E \rightarrow E$ is averaging, then $T f \in$ $L^{\infty}(\Sigma, \mu)$ and $\|T f\|_{\infty} \leq\|f\|_{\infty}$ for all $f \in L^{\infty}(\Sigma, \mu)$.

Proof. If $f \in L^{\infty}(\Sigma, \mu)$ and $\|f\|_{\infty} \leq 1$, then $(T f)^{2}=T(f T f)$ so that $(T f)^{2} \in E$ and

$$
\left\|(T f)^{2}\right\|_{E}=\|T(f T f)\|_{E} \leq\|f T f\|_{E} \leq\|f\|_{\infty}\|T f\|_{E} \leq\|f\|_{E} .
$$

Proceeding inductively, it follows that $(T f)^{n} \in E$ and $\left\|(T f)^{n}\right\|_{E} \leq$ $\|f\|_{E}$ holds for each $n=1,2, \ldots$. If $\varepsilon>0$ is given, let $A=\{\omega \in$ $\Omega:|T f(\omega)| \geq 1+\varepsilon\}$. From $|T f|^{n} \geq(1+\varepsilon)^{n} \mathbf{1}_{A}$, it follows that

$$
\left\|\mathbf{1}_{A}\right\|_{E} \leq(1+\varepsilon)^{-n}\left\|(T f)^{n}\right\|_{E} \leq(1+\varepsilon)^{-n}\|f\|_{E}, \quad n=1,2, \ldots
$$

and hence $\mu(A)=0$.

The following result is well-known and for $L^{\infty}$-spaces is a simple exercise. A simple proof for $L^{1}$-spaces may be found in [S], Lemma 5.

LEMMA 4.2. (a) If $T$ is a contraction on $L^{\infty}(\Sigma, \mu)$ and if $T \mathbf{1}=\mathbf{1}$, then $T \geq 0$.

(b) If $T$ is a contraction on $L^{1}(\Sigma, \mu)$ and if $T \mathbf{1}=\mathbf{1}$, then $T \geq 0$ and $T^{*} \mathbf{1}=\mathbf{1}$.

A direct application of Proposition 3.1 and Lemma 4.1 now yields the first part of the following result. 
Proposition 4.3. If $T: E \rightarrow E$ is an averaging contraction for which $\left\|T f_{n}\right\|_{1} \rightarrow 0$ whenever $\left\{f_{n}\right\}$ is an order bounded sequence in $E$ which converges to zero almost everywhere, then there exists a $\sigma$-subalgebra $\Sigma_{0}$ of $\Sigma$ and a function $w \in E^{\prime}$ such that $T f=\mathscr{E}\left(w f \mid \Sigma_{0}\right)$ for all $f \in E$. If in addition $T$ preserves 1 then $T \geq 0$ and $T^{2}=T$.

The second part of the proposition follows by noting that the restriction $T_{\infty}$ of $T$ to $L^{\infty}(\Sigma, \mu)$ is an $L^{\infty}$-contraction (Lemma 4.1) which preserves 1 and so $T_{\infty} \geq 0$ (Lemma $4.2(\mathrm{a})$ ). The continuity condition now implies that $T \geq 0$. That $T^{2}=T$ is an immediate consequence of the fact that $T$ is averaging and preserves 1 .

As noted earlier (see Remark 3.2 (i)), the continuity condition of the preceding proposition is satisfied if $E$ has order continuous norm. In particular the proposition holds if $E=L^{p}(\Sigma, \mu), 1 \leq p<\infty$ with $\mu(\Omega)=1$, which is the setting considered by Rota. In this special case, it may be shown that $w=1$. That this need not be the case in general is illustrated by the next example.

EXAMPLE 4.4. Let $\Omega=[0,1]$ with Lebesgue measure $\mu$. The measure $\nu$ is defined by $d \nu=w d \mu$ where $w=\frac{1}{2} \mathbf{1}_{[0,1 / 2]}+\frac{3}{2} \mathbf{1}_{(1 / 2,1]}$. Let $E$ be the Banach function space $L^{p}(\Sigma, \nu), 1 \leq p<\infty$ with the function norm

$$
\|f\|_{E}=\left(\int_{0}^{1}|f|^{p} d \nu\right)^{1 / p}, \quad f \in E,
$$

and let $\Sigma_{0}=\{\phi,[0,1]\}$. The operator $T: E \rightarrow E$ defined by $T f=$ $\mathscr{E}^{\mu}\left(w f \mid \Sigma_{0}\right)=\mathscr{E}^{\nu}\left(f \mid \Sigma_{0}\right)$ for all $f \in E$ satisfies the conditions of Proposition 4.3.

As is noted in Proposition 4.3, an averaging contraction on the Banach function space $E$ which satisfies the given continuity condition and which preserves 1 is necessarily a positive order continuous contractive projection which preserves $\mathbf{1}$. We turn now to the question of characterizing such operators in terms of conditional expectation. We recall that a Banach lattice $F$ is said to have strictly monotone norm if whenever $x, y \in F$ satisfy $0 \leq x<y$, it follows that $\|x\|_{F}<\|y\|_{F}$. We shall need the following simple result.

LEMMA 4.5. If the Banach lattice $F$ has strictly monotone norm, and if $T$ is a positive contractive projection on $F$, then the range of $T$ is a sublattice. 
Proof. If $x \in F$ and $T x=x$ then $x^{+} \leq T\left(x^{+}\right)$and so $\left\|x^{+}\right\|_{F} \leq$ $\left\|T\left(x^{+}\right)\right\|_{F} \leq\left\|x^{+}\right\|_{F}$. From strict monotonicity of the norm it follows that $T\left(x^{+}\right)=x^{+}$.

We remark that the example following Proposition 3.3 shows that the range of a positive contractive projection on a Banach lattice need not be a sublattice if the norm is not strictly monotone. In fact, in this example, one need only equip $\mathbf{R}^{3}$ with the $\|\cdot\|_{\infty}$-norm.

The following basic characterization of conditional expectation on $L^{1}(\Sigma, \mu)$ is due to R. G. Douglas [D1] and independently by G. L. Seever [S]. It is an immediate consequence of Proposition 3.3 via Lemma 4.2 (b) and Lemma 4.5.

Proposition 4.6 (Douglas, Seever). If $T$ is a continuous linear map on $L^{1}(\Sigma, \mu)$ then the following statements are equivalent.

(a) There exists a $\sigma$-subalgebra $\Sigma_{0}$ of $\Sigma$ such that $T f=\mathscr{E}\left(f \mid \Sigma_{0}\right)$ for all $f \in L^{1}(\Sigma, \mu)$.

(b) $T$ is a contractive projection which preserves $\mathbf{1}$.

We now extend this characterization to an arbitrary ideal $E$ of $L^{0}(\Sigma, \mu)$. The result which follows is a certain sharpening of Proposition 3.3. If $0 \leq T: E \rightarrow E$ is an order continuous linear map, we denote by $T^{\prime}: E^{\prime} \rightarrow E^{\prime}$ the dual mapping defined in the usual way.

Proposition 4.7. If $T: E \rightarrow E$ is a linear map, then the following statements are equivalent.

(a) There exists a $\sigma$-subalgebra $\Sigma_{0}$ of $\Sigma$ such that $T f=\mathscr{E}\left(f \mid \Sigma_{0}\right)$ for all $f \in E$.

(b) $T$ is a positive order continuous projection such that $T$ and $T^{\prime}$ preserve 1.

Proof. We need only show that (b) $\Rightarrow$ (a). An argument of Ando [A] shows that $T$ is contractive for the $L^{1}$-norm. In fact, if $f \in E$, then

$$
\begin{aligned}
\int_{\Omega}|T f| d \mu & =\int_{\Omega} T f \operatorname{sgn} T f d \mu \\
& \leq \int_{\Omega}|f| T^{\prime}(|\operatorname{sgn} T f|) d \mu \leq \int_{\Omega}|f| T^{\prime} \mathbf{1} d \mu=\int_{\Omega}|f| d \mu .
\end{aligned}
$$


Since $E$ is dense in $L^{1}(\Sigma, \mu)$ for the $L^{1}$-norm, the assertion now follows from Proposition 4.6.

For the case that $E=L^{\infty}(\Sigma, \mu)$ the preceding Proposition 4.7 reduces to [Ro], Proposition 5. To see that Proposition 4.7 yields extensions of [Ro], Theorem 1 (see also [Du] Theorem II 5.5), some further considerations are necessary. We recall the following notions. Let $\left(X,\|\cdot\|_{X}\right)$ be a real Banach space. The functional $\varphi \in X^{*}$ is called norming at the point $0 \neq x \in X$ if $\langle x, \varphi\rangle=\|x\|_{X}$ and $\|\varphi\|_{X^{*}}=1$. If such a norming functional is unique, then the norm is called smooth at the point $x$. As is well known, this is equivalent to Gâteauxdifferentiability of the norm at the point $x$ (see, for example, [Di], Chapter 2). The norm $\|\cdot\|_{X}$ is called smooth if $\|\cdot\|_{X}$ is smooth at each non-zero point of $X$. The following remark is implicit in the proof of [A], Lemma 1.

LEMMA 4.8. Suppose that $T$ is a contractive projection on the Banach space $X$ and that $x \in X$ lies in the range of $T$. If the norm is smooth at $x$ and if $\varphi \in X^{*}$ is the (unique) norming functional at $x$, then $T^{*} \varphi=\varphi$.

Proof. Since $\left\|T^{*} \varphi\right\|_{X^{*}} \leq 1$ and since

$$
\left\langle x, T^{*} \varphi\right\rangle=\langle T x, \varphi\rangle=\langle x, \varphi\rangle=\|x\|_{X}
$$

it follows that $T^{*} \varphi$ is also norming at $x$ and consequently $T^{*} \varphi=\varphi$.

We now assume that $\left(E,\|\cdot\|_{E}\right)$ is a Banach function space as considered earlier. It is not difficult to see that a constant function is norming at $\mathbf{1}$ if and only if $\|\mathbf{1}\|_{E}\|\mathbf{1}\|_{E^{\prime}}=\mu(\Omega)$. We then obtain the following consequence of Proposition 4.7.

Corollary 4.9. Suppose that the norm on $E$ is smooth at 1 and that $\|\mathbf{1}\|_{E}\|\mathbf{1}\|_{E^{\prime}}=\mu(\Omega)$. If $T$ is an order continuous positive contractive projection on $E$ which preserves 1 , then there exists a $\sigma$-subalgebra $\Sigma_{0}$ of $\Sigma$ such that $T f=\mathscr{E}\left(f \mid \Sigma_{0}\right)$ for all $f \in E$.

It will be shown in Proposition 4.16 below that if $E$ is assumed to have order continuous norm then the positivity assumption on $T$ can be replaced by the condition that the range of $T$ is a sublattice. 
It follows from Example 4.4 via a simple computation that the condition $\|\mathbf{1}\|_{E}\|\mathbf{1}\|_{E^{\prime}}=\mu(\Omega)$ cannot be omitted from the preceding Corollary 4.9. We remark that the Corollary applies immediately to the case that $E=L^{p}(\Sigma, \mu), 1 \leq p<\infty$. In this special case, the resulting characterization of positive contractive projections on $L^{p}(\Sigma, \mu)$ is due to T. Ando [A] and is itself an extension of [Ro], Theorem 1, referred to earlier. It should be mentioned that Ando ([A], Theorem 1) has proved somewhat more in this special setting: each contractive projection on $L^{p}(\Sigma, \mu)$ which preserves 1 is contractive with respect to the $L^{1}$-norm, provided $1 \leq p<\infty$ and $p \neq 2$ and hence is necessarily positive. While it does not appear that there is an immediate extension of this result to the more general setting, we consider in some detail the related question as to what extent a contractive projection onto a sublattice is positive, which is of independent interest.

First we make some remarks concerning smoothness of the norm in Banach lattices. Let $F$ be a Banach lattice. For any $0 \leq u \in F$ there exists a positive norming functional $\varphi$ which vanishes on $\{u\}^{d}$. Indeed, if $\psi \in F^{*}$ is norming at $u$, then we can take for $\varphi$ the minimal positive extension of the restriction of $|\psi|$ to the ideal in $F$ generated by $u$ (see $[\mathrm{Z}], \S 83$ ). In particular, if the norm in $F$ is smooth at $u$, then the norming functional at $u$ is positive and vanishes on $\{u\}^{d}$. Furthermore, it is worthwhile to note that a Dedekind $\sigma$-complete Banach lattice $F$, with smooth norm, necessarily has order continuous norm. Indeed, suppose on the contrary that the norm in $F$ is not order continuous. Then $F$ contains a closed subspace which is norm isomorphic to $l_{\infty}$ (see e.g. [Sch], II, 5.14). This however, is contradictory to a result of M. M. Day [Da] which states that $l_{\infty}$ cannot be renormed smoothly.

Proposition 4.10. Any contractive projection $T$ onto a sublattice of a Banach lattice $F$ with smooth norm is positive.

Proof. Take $0 \leq x \in F$ and suppose that $(T x)^{-}>0$. Let $\varphi$ be the norming functional at $(T x)^{-}$. Then $\varphi \geq 0$ and $\varphi$ vanishes on $\left\{(T x)^{-}\right\}^{d}$, so $\left\langle(T x)^{+}, \varphi\right\rangle=0$. Furthermore since the range of $T$ is a sublattice, $(T x)^{-}$is a fixed point of $T$, and hence $T^{*} \varphi=\varphi$. Therefore,

$$
0 \leq\langle x, \varphi\rangle=\left\langle x, T^{*} \varphi\right\rangle=\langle T x, \varphi\rangle=-\left\langle(T x)^{-}, \varphi\right\rangle\langle 0,
$$

a contradiction. Hence $x \geq 0$ implies that $T x \geq 0$. 
EXAMPLE 4.11. We give an example of a contractive projection onto a sublattice of a Banach lattice which is not positive. Let $B$ be the rhombic dodecahedron (see e.g. [C], §2.7) in $\mathbf{R}^{3}$ which is the absolute convex hull of the points

$$
\left\{\left(\begin{array}{l}
2 \\
0 \\
0
\end{array}\right),\left(\begin{array}{l}
0 \\
2 \\
0
\end{array}\right),\left(\begin{array}{l}
0 \\
0 \\
2
\end{array}\right),\left(\begin{array}{l}
1 \\
1 \\
1
\end{array}\right),\left(\begin{array}{c}
1 \\
-1 \\
1
\end{array}\right),\left(\begin{array}{c}
-1 \\
-1 \\
1
\end{array}\right),\left(\begin{array}{c}
-1 \\
1 \\
1
\end{array}\right)\right\} .
$$

Let $E=\mathbf{R}^{3}$ with coordinatewise ordering and define the norm $\|\cdot\|_{E}$ to be the Minkowski functional of $B$. Then $\left(E,\|\cdot\|_{E}\right)$ is a Banach lattice, but the norm is not smooth. Considering $E$ as a Banach function space on the three point measure space $\Omega$, each of the points having measure $\frac{1}{3}$, we have $\|\cdot\|_{1} \leq\|\cdot\|_{E} \leq\|\cdot\|_{\infty}$, and hence $\|\cdot\|_{1} \leq\|\cdot\|_{E^{\prime}} \leq\|\cdot\|_{\infty}$. Therefore, $\|\mathbf{1}\|_{E}=\|\mathbf{1}\|_{E^{\prime}}=1$, and thus $\|\mathbf{1}\|_{E} \cdot\|\mathbf{1}\|_{E^{\prime}}=\mu(\Omega)$. Note furthermore that $\|\cdot\|_{E}$ is rearrangement invariant.

Let $T$ be the projection onto the plane $x_{1}=x_{2}$ along $(-1,1,1)$. It is easy to verify that $T$ is a contractive projection with respect to $\|\cdot\|_{E}$. Furthermore, $T \mathbf{1}=\mathbf{1}$ and the range of $T$ is a sublattice of $E$, but $T$ is not positive.

In certain spaces in which the norm is not smooth, there is still an abundance of points at which the norm is smooth. For instance, in the space $L^{1}(\Sigma, \mu)$ the norm is smooth at all functions $f$ with $f(\omega) \neq 0$ a.e. In such cases it is sometimes possible to conclude that a contractive projection onto a sublattice is positive. The details follow. The next lemma will be useful.

LEMMA 4.12. If $F$ is a Dedekind $\sigma$-complete Banach lattice and if the norm is smooth at $0<u \in F$ then the norm is smooth at all points $x \in F$ for which $|x|=u$.

Proof. If $x \in F$ and $|x|=u$, let $P$ be the band projection in $F$ onto the band generated by $x^{-}$. If $\pi=I-2 P$ then $P u=x^{-}$implies that $\pi u=x$. Further, $|\pi z|=|z|$ for all $z \in F$ and $\pi^{2}=I$ and so $\pi$ is an isometry of $F$ onto itself. Hence $\pi$ induces a one-one correspondence between the norming functionals at $x$ and $u$ and from this the result follows.

Proposition 4.13. Let $F$ be a Banach lattice with order continuous norm and let $T$ be a contractive projection in $F$ onto a sublattice. If 
the range $R(T)$ contains weak units of $F$ and if the norm is smooth at all such weak units then $T \geq 0$.

Proof. Observe first that if $0<v, w \in R(T)$, then it is a simple consequence of the order continuity of the norm on $F$ that the component $w_{1}$ of $w$ in the principal band generated by $v$ is again in $R(T)$ since $w_{1}=\sup _{n}(w \wedge n v)$. Suppose now that $0<v \in R(T)$. We claim that there exists a weak unit $u \in R(T)$ such that the norming functional $\varphi$ at $u$ satisfies $\langle v, \varphi\rangle>0$. In fact, let $0<w \in R(T)$ be any weak unit with $\|w\|_{F}=1$. Let $w_{1}$ be the component of $w$ in the principal band generated by $v$ and set $w=w_{1}+w_{2}$. If $0<\varepsilon<\left\|w_{1}\right\|_{F}$, define $u=\varepsilon w_{2}+w_{1}$. It is clear that $u$ is a weak unit in $F$ and from the opening observation it follows that $0<u \in R(T)$. If $\varphi$ is the norming functional at $u$ then

$$
\left\|w_{1}\right\| \leq\left\|\varepsilon w_{2}+w_{1}\right\| \leq \varepsilon+\left\langle w_{1}, \varphi\right\rangle
$$

so that $\left\langle w_{1}, \varphi\right\rangle>0$. Since $0 \leq \varphi$ is order continuous and since $w_{1}=$ $\sup _{n}(w \wedge n v)$ it follows also that $\langle v, \varphi\rangle>0$ which proves the claim.

Suppose now that $0 \leq x \in F$. If $(T x)^{-}>0$, then there exists a weak unit $0 \leq u \in R(T)$ with norming functional $0 \leq \varphi \in F^{*}$ such that $\left\langle(T x)^{-}, \varphi\right\rangle>0$. Since the range of $T$ is a sublattice, it follows that $T^{*} \varphi=\varphi$. Let $P$ denote the band projection in $F$ onto the principal band generated by $(T x)^{-}$and let $\tilde{u}=(2 P-I) u$. Since $|\tilde{u}|=u$, it follows from Lemma 4.12 above that the norm is smooth at $\tilde{u}$ and moreover the functional $\tilde{\varphi}=\left(2 P^{*}-I\right) \varphi$ is norming at $\tilde{u}$. Since $u \in R(T)$, it follows also that $\tilde{u} \in R(T)$ and hence $T^{*} \tilde{\varphi}=\tilde{\varphi}$.

It now follows that $T^{*}\left(P^{*} \varphi\right)=P^{*} \varphi$ and consequently

$$
0 \leq\left\langle x, P^{*} \varphi\right\rangle=\left\langle x, T^{*} P^{*} \varphi\right\rangle=\langle P T x, \varphi\rangle=-\left\langle(T x)^{-}, \varphi\right\rangle<0
$$

and this is clearly a contradiction. Thus $0 \leq x \in F$ implies $T x \geq 0$ and the proof is complete.

A combination of Proposition 3.6 with the preceding result yields the following corollary.

COROLlARY 4.14. Let $E$ be a Banach function space with order continuous norm and let $T$ be a contractive projection in $E$ onto a sublattice. If the range of $T$ contains strictly positive functions and if the norm is smooth at all such strictly positive functions then there exists a $\sigma$-subalgebra $\Sigma_{0}$ of $\Sigma, 0 \leq w \in E^{\prime}$ and a strictly positive function $0 \leq k \in L^{1}(\Sigma, \mu)$ with $\mathscr{E}\left(w k \mid \Sigma_{0}\right)=\mathscr{E}\left(k \mid \Sigma_{0}\right)=1$ such that $T f=k \mathscr{E}\left(w f \mid \Sigma_{0}\right)$ for all $f \in E$. 
In general the existence of at least one weak unit in the range of $T$ at which the norm is smooth need not imply that $T$ is positive as an inspection of Example 4.11 shows. On the other hand, the proof of Proposition 4.13 shows that the following variant holds.

Proposition 4.15. Let $F$ be a Banach lattice with order continuous norm and let $T$ be a contractive projection of $F$ onto a sublattice. If the range of $T$ contains a weak unit $w$ of $F$ at which the norm is smooth and for which the norming functional is strictly positive, then $T \geq 0$.

The final result of the paper now follows by combining Proposition 4.15 with Corollary 4.9.

Proposition 4.16. Let $E$ be a Banach function space with order continuous norm such that the norm is smooth at $\mathbf{1}$ and such that $\|\mathbf{1}\|_{E}\|\mathbf{1}\|_{E^{\prime}}=\mu(\Omega)$. If $T$ is a contractive projection in $E$ onto a sublattice containing 1 , then there exists a $\sigma$-subalgebra $\Sigma_{0}$ of $\Sigma$ such that $T f=\mathscr{E}\left(f \mid \Sigma_{0}\right)$ for all $f \in E$.

Acknowledgments. Work on this paper by the second author was supported by a short term visiting research fellowship at Flinders University. The work of the third author was supported by A.R.G.S. and by the Netherlands Organization for the Advancement of Pure Research (Z.W.O.).

Added in proof. After completing our paper it was pointed out to us by A. V. Buhvalov that Proposition 3.3 and Corollary 3.4 also occur in some recent work of A. A. Mekler from Leningrad.

\section{REFERENCES}

[A] T. Ando, Contractive projections in $L_{p}$-spaces, Pacific J. Math., 17 (1966), 391-405.

[AB] D. D. Aliprantis and O. Burkinshaw, Positive Operators, Academic Press, Orlando, 1985.

[BL] S. J. Bernau and H. Elton Lacey, The range of a contractive projection on an $L_{p}$-space, Pacific J. Math., 53 (1974), 21-41.

[C] H. S. M. Coxeter, Regular Polytopes, Macmillan Mathematical paperbacks, New York, 1963.

[Da] M. M. Day, Strict convexity and smoothness of normed spaces, Trans. Amer. Math. Soc., 70 (1955), 516-528.

[Di] J. Diestel, Geometry of Banach Spaces, Selected Topics, Lecture notes in Mathematics, 485, Springer, Berlin, 1975. 
[D1] R. G. Douglas, Contractive projections on an $L_{1}$-space, Pacific J. Math., 15 (1965), 443-462.

[D2] _ On lattices and algebras of real valued functions, Amer. Math. Monthly, 72 (1965), 642-643.

[Du] C. V. Duplissey, Contractive projections in abstract Banach function spaces, Thesis, Austin (Texas), 1971.

[HP1] C. B. Huijsmans and B. de Pagter, Subalgebras and Riesz subspaces of an $f$-algebra, Proc. London Math. Soc., 48 (1984), 61-74.

[HP2] _ Averaging operators and positive contractive projections, J. Math. Anal. Appl., 113 (1986), 163-184.

[IT] A. and C. Ionescu Tulcea, Topics in the Theory of Lifting, Ergebnisse der Mathematik 48, Springer, Berlin, 1969.

[dJ] E. de Jonge, Conditional expectation and ordering, Ann. Probab., 7 (1979), 179-183.

[K] J. L. Kelley, Averaging operators on $C_{\infty}(X)$, Illinois J. Math., 2 (1958), 214223.

[Ku] V. G. Kulakova, Positive projections on symmetric $K B$-spaces, Proc. Steklov Inst. Math., 155 (1981); English translation (1983), 93-100.

[LZ] W. A. J. Luxemburg and A. C. Zaanen, Riesz Spaces I, North-Holland, Amsterdam, 1971.

[M] Shu-Teh Chen Moy, Characterizations of conditional expectations as a transformation on function spaces, Pacific J. Math., 4 (1954), 47-64.

[N] J. Neveu, Calcul des Probabilités, Masson, Paris, 1964.

[Ra1] M. M. Rao, Conditional expectations and closed projections, Indag. Math., 27 (1965), 100-112.

[Ra2] _ Linear operations, tensor products and contractive projections in function spaces, Studia Math., 38 (1970), 131-186.

[Ro] G. C. Rota, On the representation of averaging operators, Rend. Math. Padova, 30 (1960), 52-64.

[Sch] H. H. Schaefer, Banach Lattices and Positive Operators, Grundlehren der Mathematischen Wissenschaften 215, Springer, Berlin, 1974.

[S] G. L. Seever, Nonnegative projections on $C_{0}(X)$, Pacific J. Math., 17 (1966), 159-166.

[Z] A. C. Zaanen, Riesz Spaces II, North-Holland, Amsterdam 1983.

Received February 19, 1988.

The Flinders University of South Australia

BedFord Park, S.A. 5042, Australia

UNIVERSITY OF LEIDEN

NiELS BOHRWEG 1

P.O. Box 9512

2300 R.A. LEIDEN, NETHERLANDS

AND

Delft University of TeChNOLOGY

JULIANALAAN 132

2628 BL DELFT, Netherlands 



\section{PACIFIC JOURNAL OF MATHEMATICS EDITORS}

\author{
V. S. VARADARAJAN \\ (Managing Editor) \\ University of California \\ Los Angeles, CA 90024-1555-05 \\ Herbert Clemens \\ University of Utah \\ Salt Lake City, UT 84112 \\ ThOMAS ENRIGHT \\ University of California, San Diego \\ La Jolla, CA 92093
}

R. FINN

Stanford University

Stanford, CA 94305

HeRmann FlaschKa

University of Arizona

Tucson, AZ 85721

VAUGHAN F. R. JONES

University of California

Berkeley, CA 94720

Steven KerckHofF

Stanford University

Stanford, CA 94305
RoBION KIRBY

University of California

Berkeley, CA 94720

C. C. MOORE

University of California

Berkeley, CA 94720

Harold Stark

University of California, San Diego

La Jolla, CA 92093

\section{ASSOCIATE EDITORS}

\begin{tabular}{|c|c|c|c|c|}
\hline R. ARENS & $\begin{array}{l}\text { E. F. BECKENBACH } \\
(1906-1982)\end{array}$ & H. NeumanN & F. Wolf & K. YoshidA \\
\hline \multicolumn{5}{|c|}{ SUPPORTING INSTITUTIONS } \\
\hline \multicolumn{2}{|c|}{ UNIVERSITY OF ARIZONA } & \multicolumn{3}{|c|}{ UNIVERSITY OF OREGON } \\
\hline \multirow{2}{*}{\multicolumn{2}{|c|}{$\begin{array}{l}\text { UNIVERSITY OF BRITISH COLUMBIA } \\
\text { CALIFORNIA INSTITUTE OF TECHNOLOGY }\end{array}$}} & \multicolumn{3}{|c|}{ UNIVERSITY OF SOUTHERN CALIFORNIA } \\
\hline & & \multicolumn{3}{|c|}{ STANFORD UNIVERSITY } \\
\hline \multicolumn{2}{|c|}{ UNIVERSITY OF CALIFORNIA } & \multicolumn{3}{|c|}{ UNIVERSITY OF HAWAII } \\
\hline \multicolumn{2}{|c|}{ MONTANA STATE UNIVERSITY } & \multicolumn{3}{|c|}{ UNIVERSITY OF TOKYO } \\
\hline \multicolumn{2}{|c|}{ UNIVERSITY OF NEVADA, RENO } & \multicolumn{3}{|c|}{ UNIVERSITY OF UTAH } \\
\hline \multicolumn{2}{|c|}{ NEW MEXICO STATE UNIVERSITY } & \multicolumn{3}{|c|}{ WASHINGTON STATE UNIVERSITY } \\
\hline OREGON STA & UNIVERSITY & \multicolumn{3}{|c|}{ UNIVERSITY OF WASHINGTON } \\
\hline
\end{tabular}




\section{Pacific Journal of Mathematics}

\section{Vol. 141, No. $1 \quad$ November, 1990}

Yusuf Abu-Muhanna and Abdallah Khalil Lyzzaik, The boundary

behaviour of harmonic univalent maps $\ldots \ldots \ldots \ldots \ldots \ldots \ldots \ldots \ldots \ldots$

Lawrence Jay Corwin, Allen Moy and Paul J. Sally, Jr., Degrees and

formal degrees for division algebras and $\mathrm{GL}_{n}$ over a $p$-adic field $\ldots \ldots \ldots 21$

Ulrich Dierkes and Gerhard Huisken, The $n$-dimensional analogue of the

catenary: existence and nonexistence $\ldots \ldots \ldots \ldots \ldots \ldots \ldots \ldots 47$

Peter Gerard Dodds, C. B. Huijsmans and Bernardus de Pagter,

Characterizations of conditional expectation-type operators $\ldots \ldots \ldots \ldots 55$

Douglas Dokken and Robert Ellis, The Poisson flow associated with a

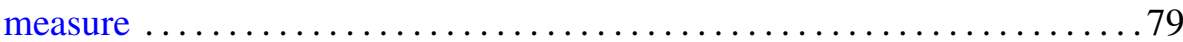

Larry J. Santoni, Horrocks' question for monomially graded modules . . . . 105

Zbigniew Slodkowski, Pseudoconvex classes of functions. II. Affine

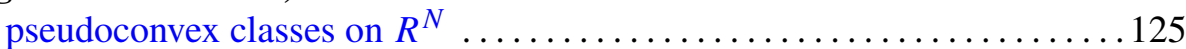

Dean Ellis Smith, On the Cohen-Macaulay property in commutative algebra

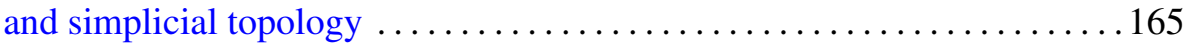

Michał Szurek and Jaroslaw Wisniewski, Fano bundles over $P^{3}$ and $Q_{3} \ldots 197$ 